\title{
Advances in LADAR Components and Subsystems at Raytheon*
}

\author{
Michael Jack, George Chapman, John Edwards, \\ William Mc Keag, Tricia Veeder, Justin Wehner \\ Raytheon Vision Systems, 75 Coromar Drive; Goleta, CA 93117 \\ Tom Roberts, Tom Robinson, James Neisz, Cliff Andressen, Robert Rinker \\ Raytheon Missile Systems, 1151 E. Hermans Rd, Tucson, AZ 85756 \\ Donald N.B. Hall hall@ifa.hawaii.edu, Shane M. Jacobson \\ Institute for Astronomy, University of Hawaii \\ Farzin Amzajerdian from NASA Langley Research Center \\ T. Dean Cook from NAWC, China Lake
}

\begin{abstract}
Raytheon is developing NIR sensor chip assemblies (SCAs) for scanning and staring 3D LADAR systems. High sensitivity is obtained by integrating high performance detectors with gain, i.e., APDs with very low noise Readout Integrated Circuits (ROICs). Unique aspects of these designs include: independent acquisition (non-gated) of pulse returns, multiple pulse returns with both time and intensity reported to enable full 3D reconstruction of the image. Recent breakthrough in device design has resulted in HgCdTe APDs operating at 300K with essentially no excess noise to gains in excess of 100, low NEP $<1 \mathrm{nW}$ and GHz bandwidths and have demonstrated linear mode photon counting. SCAs utilizing these high performance APDs have been integrated and demonstrated excellent spatial and range resolution enabling detailed 3D imagery both at short range and long ranges. In the following we will review progress in real-time 3D LADAR imaging receiver products in three areas: (1) scanning $256 \times 4$ configuration for the Multi-Mode Sensor Seeker (MMSS) program and (2) staring $256 \times 256$ configuration for the Autonomous Landing and Hazard Avoidance Technology (ALHAT) lunar landing mission and (3) Photon-Counting SCAs which have demonstrated a dramatic reduction in dark count rate due to improved design, operation and processing.
\end{abstract}

Keywords: APD, HgCdTe, LADAR

\section{INTRODUCTION AND OUTLINE}

This paper provides an overview of advanced LADAR receiver technology at Raytheon Vision Systems (RVS) ranging from high sensitivity single element APDs and associated low noise amplifiers to high speed scanning arrays $256 \times 4$ and very large format $256 \times 256$ asynchronous (non-gated) flash receivers and small format linear mode photon counting modules. These have in common the use of MBE grown $n$ on p HgCdTe engineered for excess noise factors near unity, operation at ambient temperatures and bandwidth up to $1 \mathrm{GHz}$. In this paper we focus on the status of real-time 3D imaging LADAR receiver products in scanning and staring and photon counting configurations. The scanning $256 \times 4$ receiver is a 2nd generation sensor developed for the Multi-Mission Sensor Seeker (MMSS) program. The staring $256 \times$ 256 receiver, the largest HgCdTe APD LADAR receiver operating at ambient, uses a sophisticated multiple laser pulse return ROIC. Its development has been supported in part by NASA under the Autonomous Landing Hazard Avoidance Technology ALHAT program. Finally we review breakthroughs in photon counting SCAs with reduction in dark count

\footnotetext{
* The authors acknowledge support from NASA Langley under the ALHAT program (contract NNL08AA46C), program manager, Dr. Farzin Amzajerdian, from the MMSS program contract \# N68936-08-C-0034 program manager, T. Dean Cook, NAWC, (China Lake) and from NASA ROSES 2006 Grant NNX08AE07G to the University of Hawaii, Eric P. Smith, HQ Program Scientist; subcontract Z944371 to Raytheon.
} 
rate due to improvement in design, operation and processing. Work here has been supported at Raytheon by University of Hawaii, Institute for Astronomy under a grant from NASA's Research Opportunities in Space and Earth Sciences (ROSES) program.

Section 2 provides an overview of HgCdTe APD based LADAR sensor products at Raytheon with examples of performance specifications. Section 3 discusses applications, Section 4 reviews key properties and advantages of HgCdTe APDS, Section 5 provides an example of the ROICs employed in LADAR products, Section 6 discusses scanning LADAR reviews the motivation, requirements, and summarizes the integrated receiver and its performance data at SCA and Integrated Dewar Assembly (IDA) level for the MMSS $256 \times 4$ scanning LADAR sensors, Section 7 describes the ALHAT mission and flow down requirements for the $256 \times 256$ flash and reviews the ALHAT receiver's detector and ROIC subcomponents and the performance of the $256 \times 256$ integrated SCA and IDA. Section 8 reviews photon counting technology highlighting major improvements in integration time and dark count rate.. Section 9 summarizes the paper. Section 10 looks at future directions and Section 8 acknowledges support.

\section{LADAR SENSORS AT RAYTHEON}

\subsection{LADAR Products at Raytheon Vision Systems}

Figure 1 illustrates the major LADAR receiver products and components at RVS.

These are primarily based on MBE engineered HgCdTe APDs with Fex 1, high gain and GHz bandwidths at ambient temperatures.

- Large $100 \mu \mathrm{m}$ to $300 \mu \mathrm{m}$ single element APDs for ambient or TEC operation in the 1-2 $\mu \mathrm{m}$ band (1a).

- Scanning LADAR sensors $4 \times 256$ configuration (1b).

- Large format flash sensors $(256 \times 256)$ providing up to three laser pulse returns with both range and intensity (1c). This structure is used for the ALHAT sensor described in Sections 5 and 6.

- Photon counting modules with electronics and dewar for long range LADAR and laser communications (1d).

These 3D Imaging LADAR Receivers combine common elements:

- Innovative Readout Integrated Circuits with

- No Excess Noise Engineered HgCdTe APDs

To Provide Unique Advantages for LADAR Systems

- Tactical LADAR (Ambient 300K Operation)

- Photon Counting Sensors (cooled). 


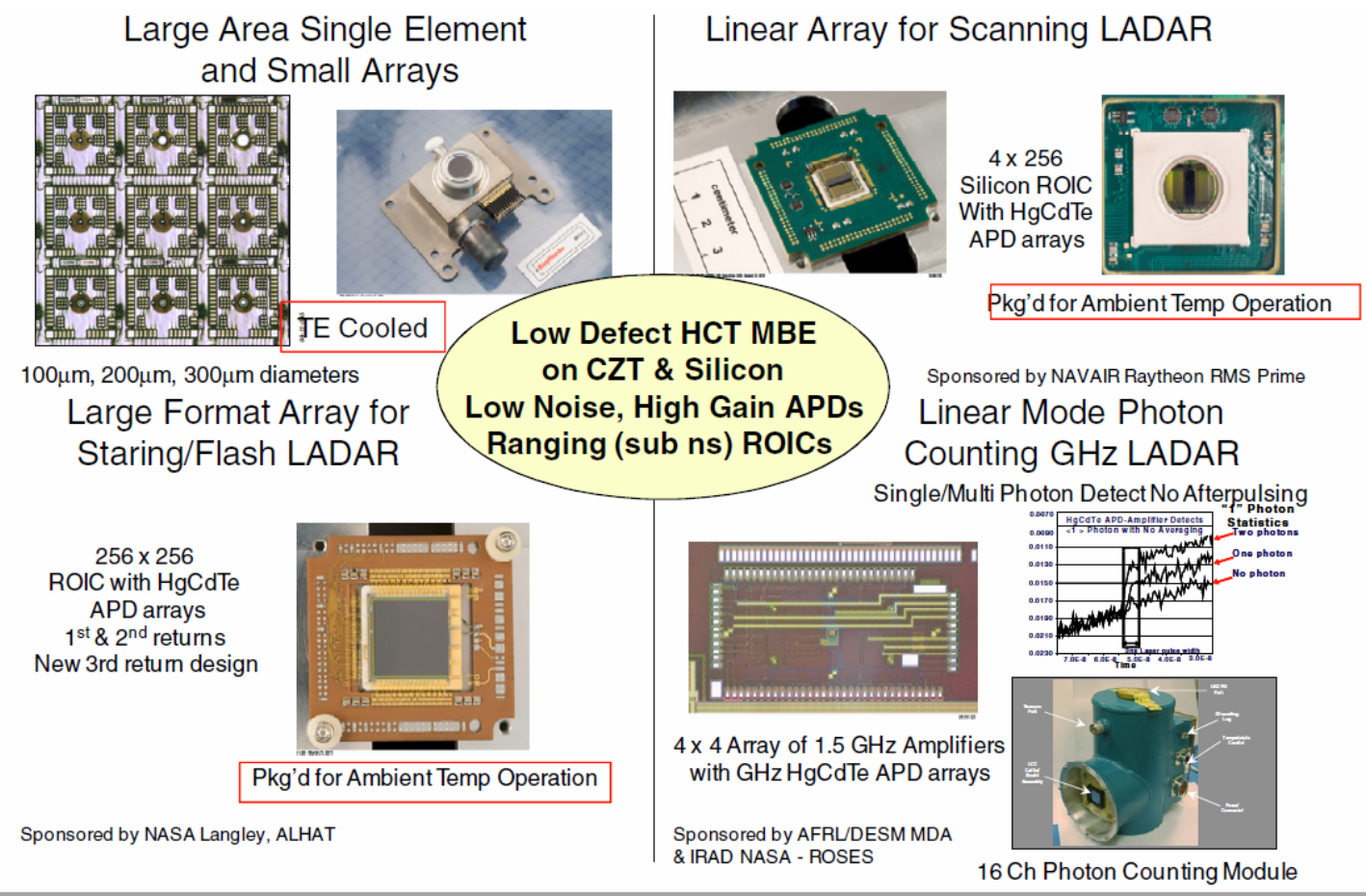

\section{Raytheon's LADAR Sensors Combine Innovative ROICs \& No Excess Noise Engineered HgCdTe APDs to Provide Unique Advantages for Tactical Ambient Temp and Photon Counting LADAR Systems}

Figure 1. LADAR Products at Raytheon include: High Performance Large $100 \mu \mathrm{m}$ to $300 \mu \mathrm{m}$ Single Element APDs for Ambient or TEC Operation in the 1-2 $\mu \mathrm{m}$ band: (2) Scanning LADAR sensors $4 \times 256$ configuration; (3) Large Format Flash Sensors $256 \times 256$ Providing up to three laser pulse returns with both range and intensity and (4) Photon Counting Modules with Electronics and Dewar.

\section{STARING, SCANNING, AND PHOTON COUNTING LADAR APPLICATIONS}

Figure 2 illustrates some of the applications this receiver technology addresses. Common to these are the combination of HgCdTe APD arrays as shown hybridized to very low noise high speed Readout Integrated Circuits. Applications include as shown (a)3D imaging at long ranges with high spatial and range resolution, (b) high confidence identification of threats on land or sea, (c) navigation and landing here for lunar or mars missions and (d) Ultra sensitive photon counting for astronomy and long range surveillance. 


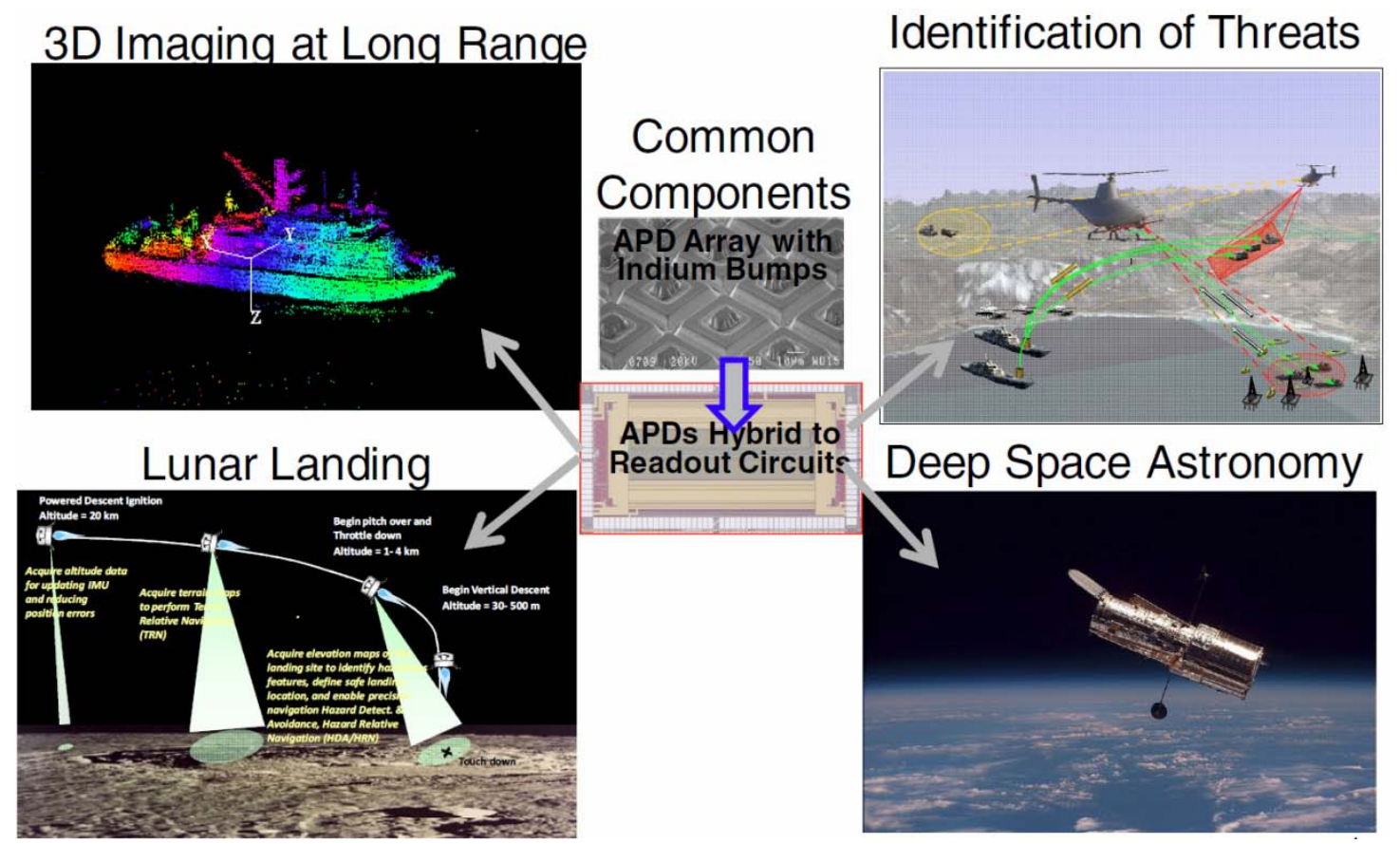

Figure 2. Core sensor technology (HgCdTe APDs and hybrids) and Applications including: (a) 3D imaging at long range, (b) high confidence threat detection, navigation and (c) landing and deep space astronomy using photon counting.

Figure 3 summarizes specific projects for which LADAR receivers have been provided.

- PROJECT: Multi-mode Sensor Seeker (MMSS)

- CUSTOMER: NAVAIR

- APPLICATION: 3D imaging for Naval fleet surveillance, reconnaissance, and target ID

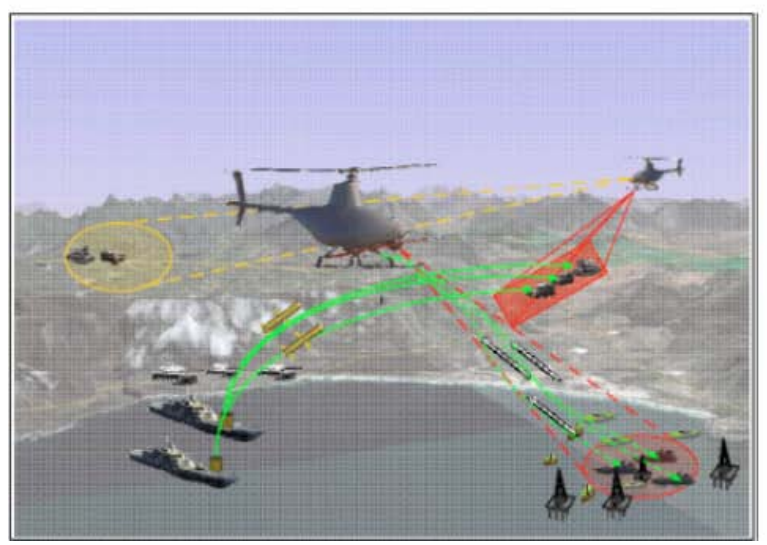

- PROJECT: Autonomous Landing and Hazard Avoidance Technology (ALHAT)

- CUSTOMER: NASA Langley

- APPLICATION: 3D imaging for lunar landing and navigation

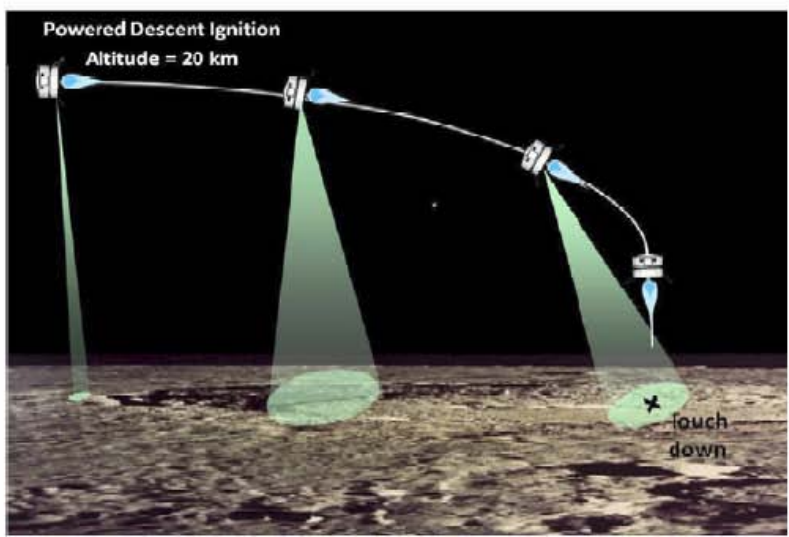

Figure 3. Raytheon is providing uncooled LADAR receivers based on common HgCdTe APD detectors and advanced ROICs for both Scanning (MMSS) Littoral Defense and Staring (ALHAT) Lunar Landing Missions 


\subsection{Real-time LADAR Imagery from 1st Generation Sensors}

Figure 4 shows the 3D images from Raytheon's 1st Generation Flash LADAR. On the top is one of many 3D images of boats. Color represents range. They were taken with a $128 \times 2$ Scanning LADAR receiver sensor which has provided excellent imagery in the field for over two years. As shown in the top and bottom (moving) images, the receiver, captures wide FOV 3D images in real-time. Operation of the sensor is asynchronous, i.e., the close and far object images are obtained with one laser pulse. Incremental time gating is not required.

口TEC Stabilized at 300K

$$
\begin{aligned}
& \text { asynchronous Not Gated } \\
& \text { Operation }
\end{aligned}
$$

DFast output enables real time imaging

aNo need for motion

\section{aRange is Color Coded}

- Green Closest

-Red Furthest

DReal-time LADAR Sequence (Vertical Axis Color Map)

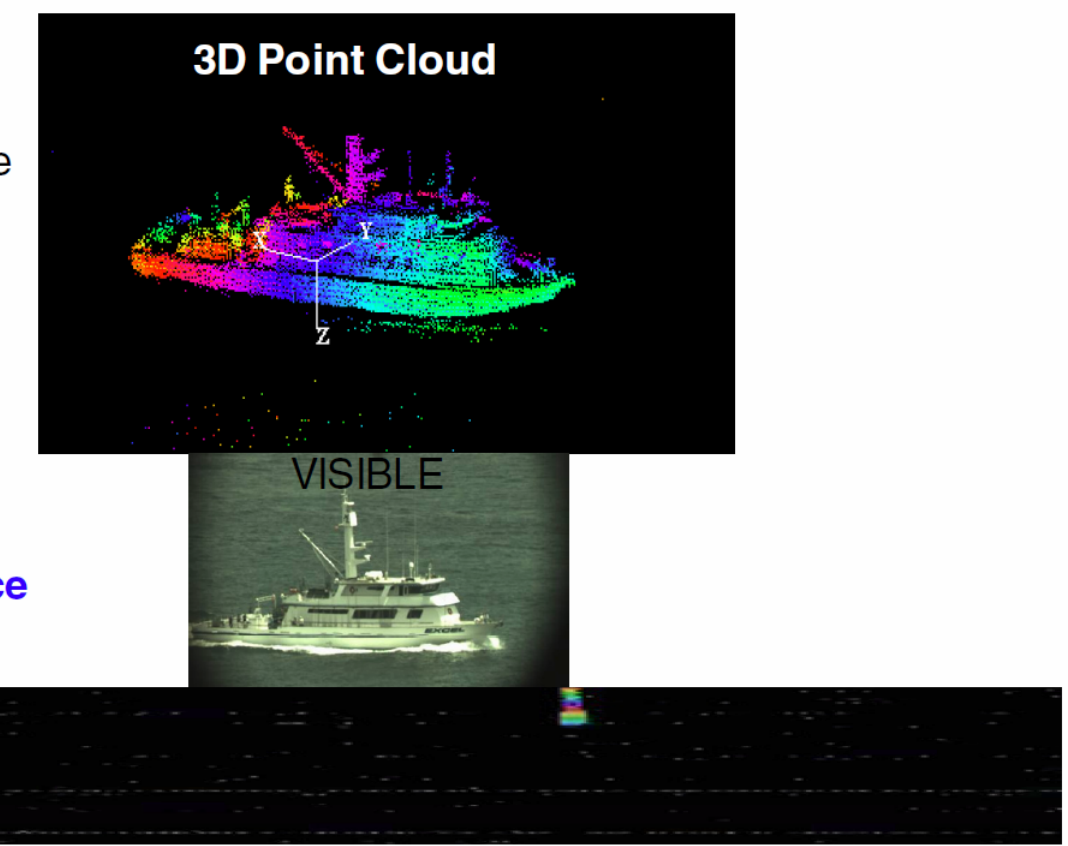

Tactical 300K LADAR Provides 3D Resolution for Long Range Object ID
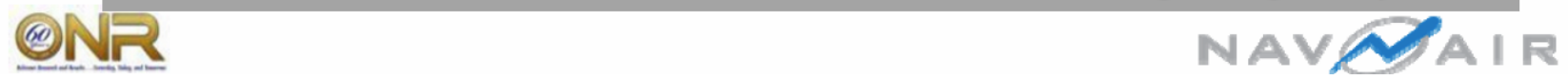

Figure 4 Navy Images from Raytheon's 1st Generation Flash LADAR (a) 3D Point Cloud Color Maps into Range; (b)

Visible Image; (c) Movie Illustrating Real-time 3D Image Capture of Boats.

\section{DETECTOR DESIGN}

For tactical ambient temperature tactical mission applications HgCdTe can be engineered to provide high gain and low excess noise at $300 \mathrm{~K}$ or higher. Under these circumstances we note the following with reference to Figure 5.

- Most APDs obey the McIntyre excess noise equation: $F e x=k_{\text {eff }} \times M_{e}+\left(2-1 / M_{e}\right) \times\left(1-k_{\text {eff }}\right)($ Fig. 5)

o $\quad k_{\text {eff }}=$ Ionization ratio of holes/Ionization rate of electrons, $M_{e}=$ Gain

- Most III-V APDS have $\mathrm{k} \sim 0.15-0.2$ resulting in Fex 10 at gain of $\mathbf{4 0}$ The theoretical best is $\mathrm{F}_{\mathrm{ex}}=2$

- HgCdTe electron injection show gain and excess noise properties indicative of deterministic single carrier gain

- Thus for HgCdTe Fex is 1 (Ideal Amplifier) is Achieved:

- Also RVS has uniquely engineered MBE HgCdTe APDs to have Fex 1 at 300K

From Figure 4 HgCdTe at Me $\sim 100$ enables 10-20 x lower noise than III-V APDs and 4x lower noise than Si. Figure 5 shows the low excess noise achieved $\mathrm{F}_{\mathrm{ex}} \sim 1$ for HgCdTe, in comparison with InAlAs and Silicon APDs. At $\mathrm{M}_{\mathrm{e}} \sim 100$ the HgCdTe shows 4x lower noise floor than Si and 20x lower noise than InAlAs APDs. 


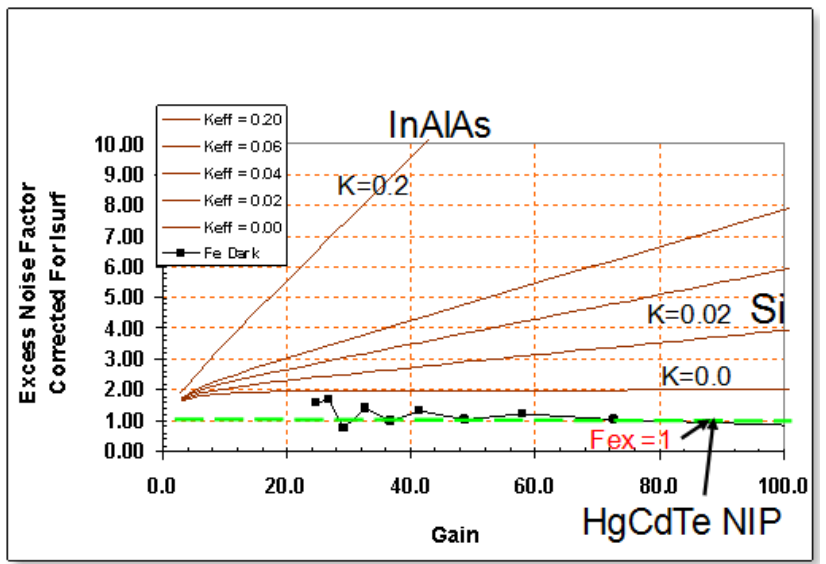

Figure 5. Excess Noise vs. Gain for HgCdTe APDs Measured vs. III-V \& Si (McIntyre Equation)

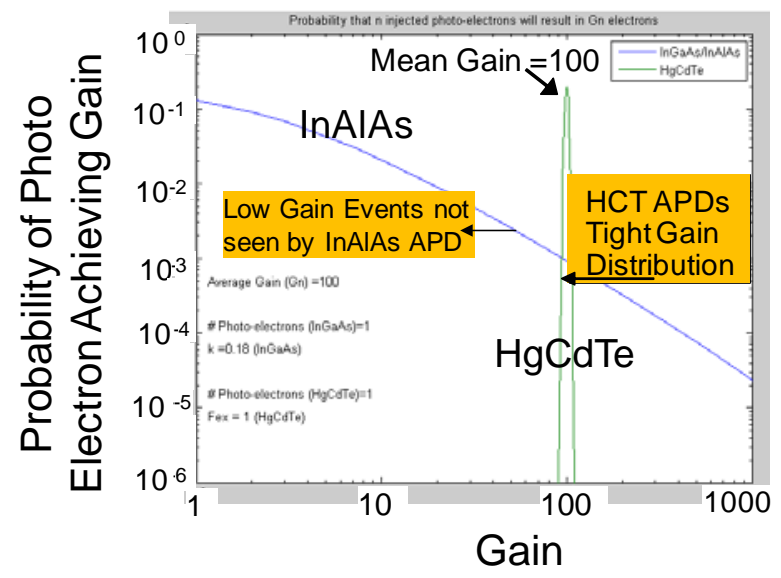

Figure 6. Probability of Electron Achieving a Gain vs. Gain. HgCdTe APD Achieve a Very Tight Gain Distribution around the average gain of 100 . InAlAs APDs $\left(\mathrm{k}_{\mathrm{eff}} \sim 0.18\right)$ have very wide gain dispersion.

\subsection{Photon Counting}

For photon counting applications excess noise, $\mathrm{F}_{\mathrm{ex}}$, reflects the variation in gain from laser shot to shot. This can be seen in Figure 6, which plots probability of achieving a gain vs. gain. The distribution of gains for HgCdTe is close to the average value here 100 . For InAlAs with $\mathrm{k} \sim 0.18$ probability of achieving the distribution of gains is very large with the highest probability at low gains and low but not zero probability at gains well above 100. These supergain events occur occasionally but skew the average so that the mean is still 100 . However if threshold is set at $1 / 2$ the average value of gain i.e. 50, we will miss over $90 \%$ of the events. So probability of detection is low. If we set a very low threshold noise will trigger the detection so we will get many false alarms.

- $\left.\mathrm{F}_{\mathrm{ex}}=\left\langle\mathrm{M}_{\mathrm{e}}^{2}\right\rangle /<\mathrm{M}_{\mathrm{e}}\right\rangle^{2} \quad$ Variance/Average

- For HgCdTe APDs $F_{\text {ex }}=1$ Implies Zero or Minimal Gain Variation from Shot to Shot (Low gain dispersion)

- Achieves Higher Probability of Detection than III-V or Silicon APDs 


\section{READOUT INTEGRATED CIRCUITS}

Figure 7 illustrates typical parameters for our asynchronous ROICs. These large format chips use advanced submicron CMOS. Each pixel independently detects a return pulse and acquires time of arrival to sub-nanosecond range precision and range accuracy as well as intensity for up to three laser returns per frame. The design shown in Figure 7 is $256 \times$ 256 the largest HgCdTe based LADAR receiver operating at ambient temperature.

\section{- SB $428 \mathrm{ROIC}^{1}$ Parameters}

$-256 \times 256$ Format

- 0.18 micron CMOS

- 60 Micron Pixels

- Asynchronous Acquisition of Laser Returns (not gated)

- Low Noise Amplification $<0.5 \mathrm{pA} / \mathrm{rt}-\mathrm{Hz}$

- Range and Intensity Acquired for up to 3 returns

- Range Precision 5cm (0.3nS)

- $30 \mathrm{~Hz}$ Operation

- ALHAT ${ }^{1}$ SCA

- Indium Bump Hybridized HgCdTe APD Arrays to SB 428 ROICs

- Forms 65536 Pixel LADAR Receiver

- Ambient Operation

- 1-2 micron spectral region

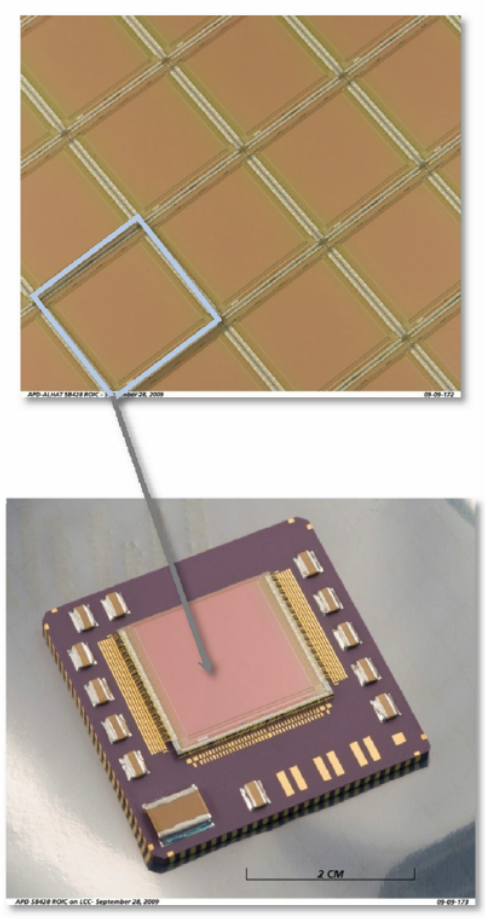

'Built for NASA's Autonomous Landing and Hazard Avoidance Technology (ALHAT)Program

\section{$256^{2}$ Asychronous Detection of 3 Returns with $5 \mathrm{~cm}$ Range Precision}

Figure 7. Low Noise High Speed ROICs Capture Multiple Returns with Sub-nanosecond Range Precision 


\section{SCANNING LADAR - MMSS PROGRAM}

\subsection{SCAs and IDAs}

Figure 8a shows the $256 \times 4$ Scanning LADAR SCA developed for the MMSS program, installed in a TEC stabilized package before sealing. The SCA uses APD array and circuit technology nearly identical to that used for the large flash array. The hermetic package utilized is compact and common to both designs. The single stage TEC enables operation in standard Mil-Spec environments. The interface board provides bypass capacitors, multiplexers and LVDS receivers. Figure 8b shows the IDA after vacuum package and sealing.

\section{- APD Detector Array \\ - Readout Integrated Circuit \\ - Platform \\ - Interface PWB \\ - Multiplexers}

- Integrated Dewar Assembly (IDA) Components Pre Top Seal:
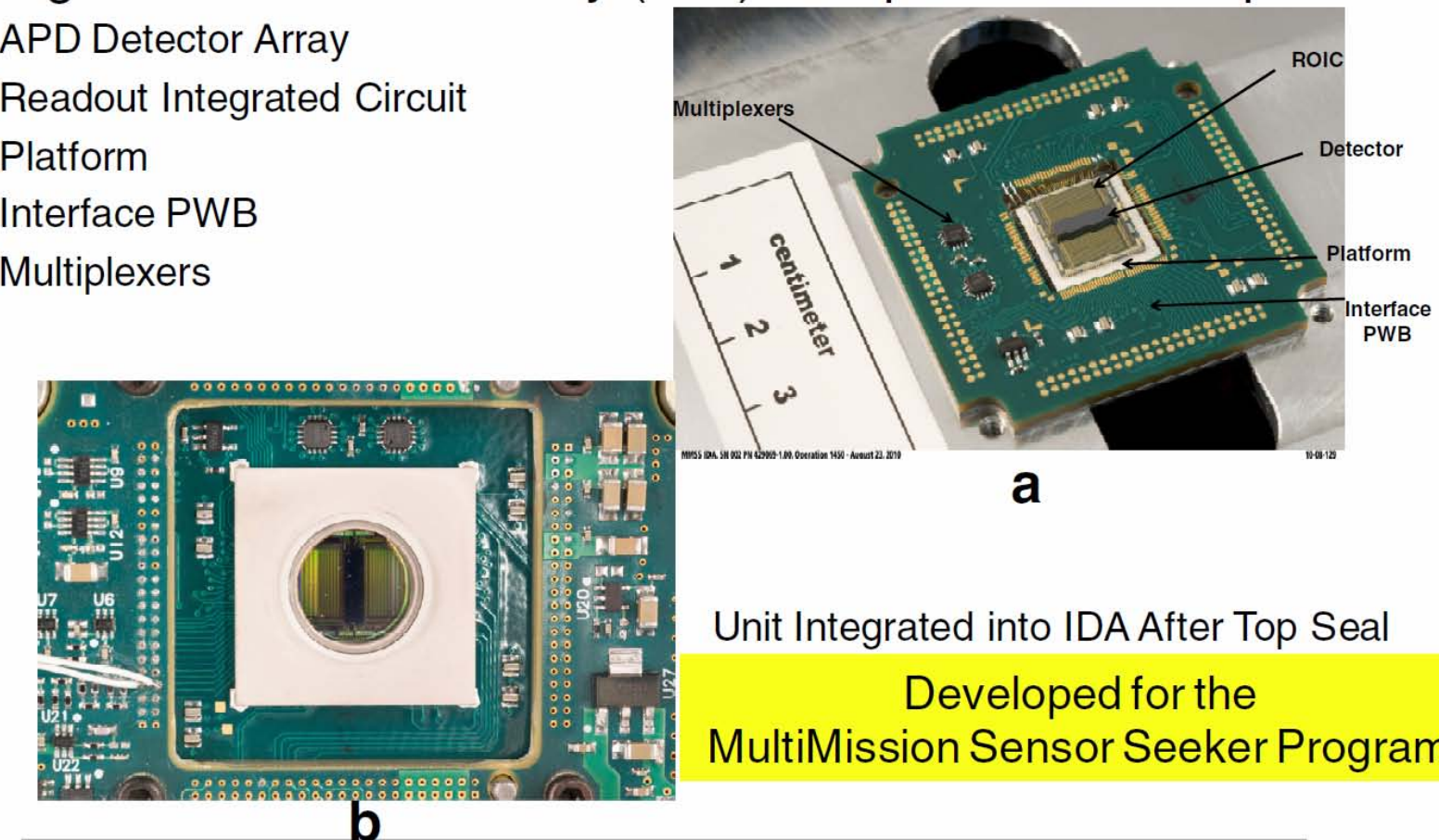

a

Unit Integrated into IDA After Top Seal

Developed for the MultiMission Sensor Seeker Program

\section{Integrated Units Tested and Delivered to Customer 1Q 2012}

Figure 8. $2^{\text {nd }}$ Gen Line Scan LADAR $256 \times 4$ SCAs Integrated into Thermal Electrically Cooled Packag

\subsection{Test Results}

Units are extensively tested both at SCA and at IDA assembly stages. Full array measurements are made of responsivity, Noise Equivalent Power (NEP) and Minimum Detectable Signal (MDS) all at ambient temperature. The latter MDS measurement is an overall determination of minimum laser power needed for high confidence detection by the full array. The measurement outcome is shown in Figure 9. MDS is measured as part of performance qualification at SCA test (and after integration into the TEC package) by uniformly illuminating the receiver chip with a pulsed laser at $1.55 \mu \mathrm{m}$. Prior to illumination pixels are adjusted to a specified false alarm rate i.e. one false alarm per 100 frames or $1 \%$. The laser then uniformly illuminates that array and the state of each pixel is measured after each pulse. If the pixel "trips" in response to the laser 95 times out of 100 pulses, the pixel is identified as good. At moderate levels of illumination nearly all the pixels will trip in response to the laser pulse. For this typical array we have 99.6\% detection operability. As the laser power is reduced some of the pixels do not respond 95 times out of 100 . The power where at least $95 \%$ of the pixels respond 95 times out of 100 shots is defined as the MDS with a specified FAR in this case $1 \%$.

The curve in Figure 9 plots the number of pixels which detect vs. flux. The MDS is then the value of the horizontal axis when the vertical axis is $95 \%$ of the array. MDS curves plotted as a function of APD gain, illustrate the dramatic 10x 
improvements as gain is increased up 20 in this case. NOTE: In all cases, the chip biases are adjusted so that no more than $1 \%$ false alarms are recorded. IDAs qualified by this final ambient temperature test were delivered to the customer $1 \mathrm{Q}$ of 2012.

\section{- MDS Improves (Reduces) with Higher APD Gains}

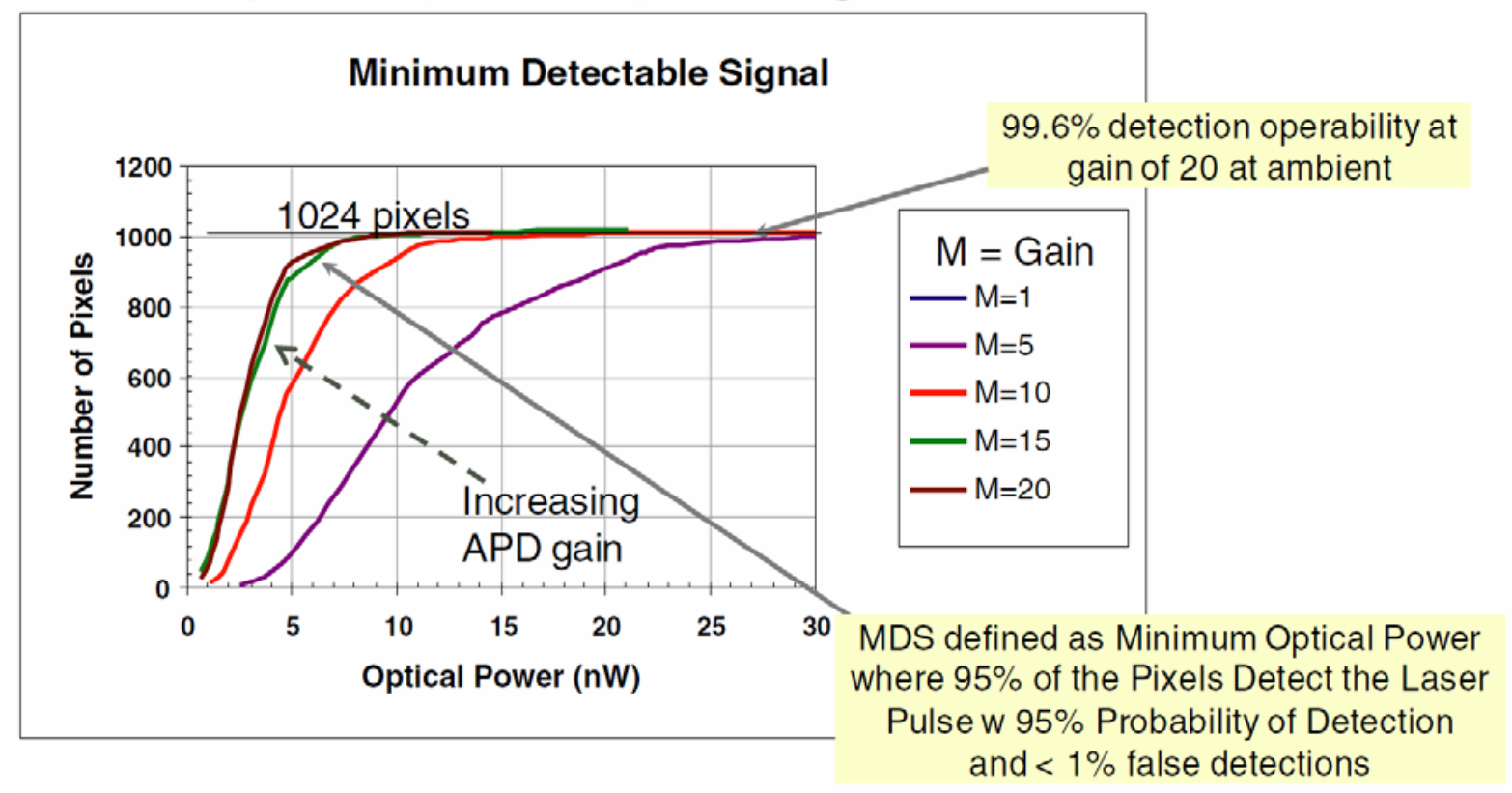

\section{MDS Measured at 300K - Units Shipped Meet Requirements}

Figure 9. Minimum Detectable Signal (MDS)Curves Qualify SCA and IDA Performance at the Array Level

\section{STARING ARRAYS - ALHAT PROJECT}

NASA 's Autonomous Precision Landing and Hazard Detection Avoidance Technology (ALHAT) project is actively pursuing development of 3-D Imaging Flash LADAR technology for precision safe landing on the Moon and other planetary bodies. Future robotic and human exploration vehicles to the Moon will require advanced technology development to support safe and precision landing on the lunar surface. Lunar exploration may require landing in areas of total darkness where hazardous terrain features such as rocks or craters could be obscured. Hazard detection must be quick, achievable under any lighting condition, and at a far enough range to divert and guide the vehicle to a safe landing zone. Although precision-landing accuracies of tens of meters will be required for these missions, the hazard detection sensor will need to resolve terrain features at a few centimeters in-depth from standoff ranges hundreds of meters away. As illustrated in Figure 10, the ALHAT project will develop descent and landing Guidance, Navigation and Control (GNC) hardware and software technologies necessary to advance the state-of-the-art in order to support lunar crew, cargo, or robotic missions.

The primary objective of ALHAT is to develop and mature an autonomous lunar landing GN\&C and sensing system meeting the following general requirements:

- Improve Landing Accuracy from $1 \mathrm{~km}$ to 30 meters

- Incorporate Hazard Detection and Avoidance capability (Detecting rocks $>30 \mathrm{~cm}$ in height, and slopes $>5$ deg. over $10 \mathrm{~m}$ )

- Enable landing anywhere and under any lighting conditions 
Although ALHAT focuses on lunar landing, its technology can be applied to landing in other planetary bodies in the solar system (e.g. Mars), Figure 10.

Flash LADAR can perform three functions critical for precision safe landing:

- Hazard Detection and Avoidance (1 km to $100 \mathrm{~m}$ )

- Terrain Relative Navigation (15 km to $2 \mathrm{~km}$ )

- Altimetry (20 km to $100 \mathrm{~m}$ )

Figure 11 provides the ALHAT Flash LADAR operational scenario and a description of these functions.

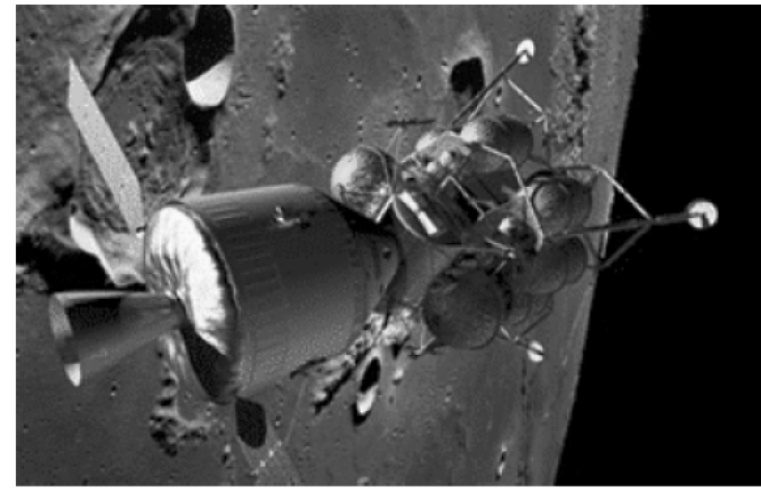

Figure 10. Lander is Shown in Close Proximity to Cratered Lunar or Planetary Surface

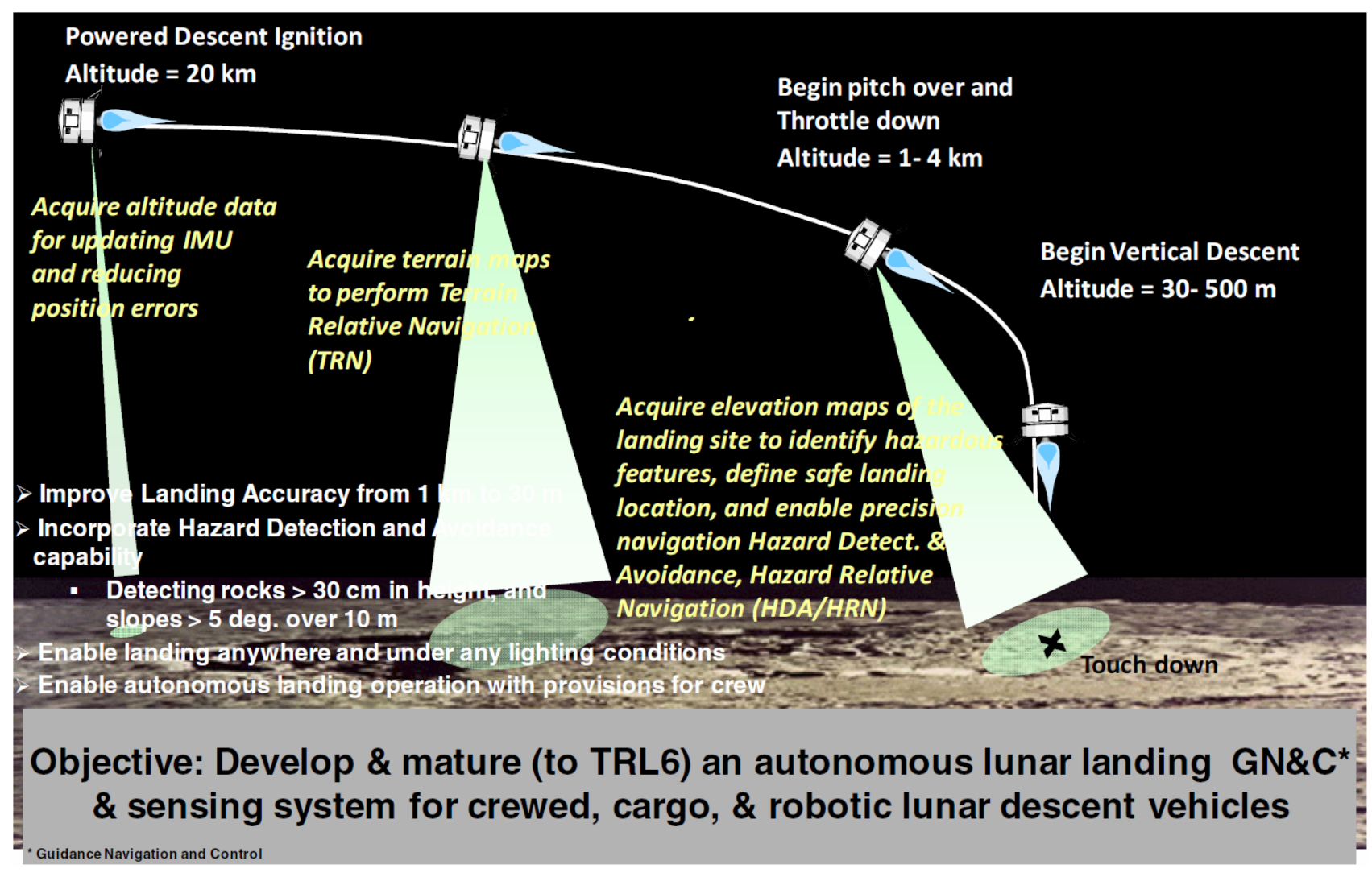

Figure 11. ALHAT Operational Scenario: 3D Flash LADAR acquires altitude data, generates terrain for navigation and acquires precise elevation information identifying hazardous features. This enables precise navigation and landing at a safe location. 


\subsection{ALHAT HgCdTe Avalanche Photodiodes}

APD layers are grown by MBE, and processed into $256 \times 256$ arrays using fabrication methods similar to those used for conventional HgCdTe IR detectors. Once fabricated, arrays are tested at wafer level for key characteristics listed below. Statistical measurements provide an early assessment of uniformity expected for the final product. Figure 12 illustrates the protocol selected for statistical measurements on the wafer. Forty five diodes were tested every fifth diode along a row that spans 256 APDs. Gain, responsivity, dark current and NEP were extracted. Figure 13 shows a skyline plot of all 45 diodes at various bias voltages selected to achieve gains of 5, 10, 20, 40, 60, 80 and 100. All APDs tested had full operability and achieved a gain of 100 . Only small spatial variations in gain are observed along the row of 45 diodes as bias is adjusted to achieve each nominal gain from 5 to 100 . Non-uniformity (sigma/mean) of $<8 \%$ was seen for all gains. With low defect wafers now being grown this excellent performance is routinely seen on multiple wafers.

- Bias dependence data taken on 45 diodes

o Detector bias voltage stepped

o Signal and noise measurements

o Test used to derive excess noise factor

- Data extracted and plotted

o Gain

o Responsivity

o Dark Current

o NEP@ $42 \mathrm{MHz}$ bandwidth

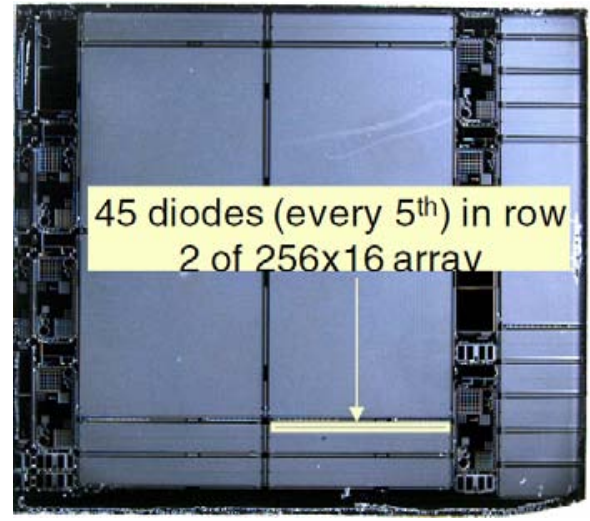

Figure 12. Statistical APD Data Derived From Probe Test of Wafer

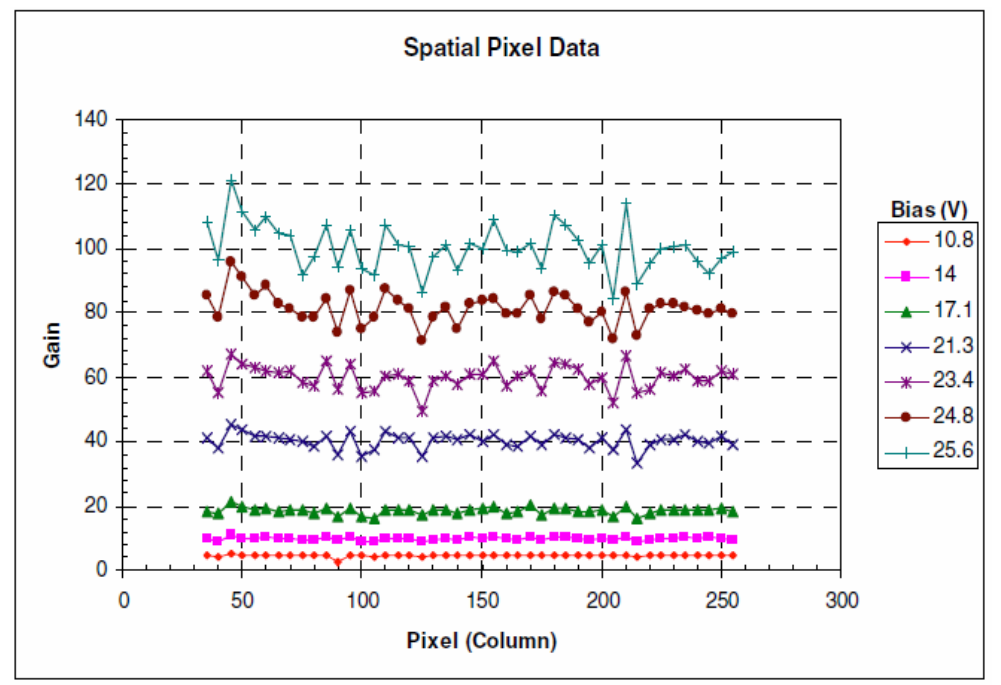

\subsection{ROICs}

The ALHAT $256 \times 256$ ROIC is a $4^{\text {th }}$ generation design. It has been optimized for performance required by NASA for the lunar landing. As discussed in Section 3.0, this design uses a $0.18 \mu \mathrm{m}$ CMOS process to provide sophisticated functionality per pixel while achieving the desired very low noise $0.5 \mathrm{pA} / \mathrm{rt}-\mathrm{Hz}$ to complement the APD performance. Laser returns are detected asynchronously (not gated) and both intensity and time of arrival are acquired for up to three 
returns per frame time. Timing precision is key to accurate relative range data, i.e., range precision. To achieve required $5 \mathrm{~cm}$ range precision the ROIC timing accuracy has been improved to 300ps. Multiple returns from a single outgoing pulse are expected when a pixel sees the edge of a boulder and the adjacent ground.

- $256 \times 256$ format

- $0.18 \mu \mathrm{m}$ CMOS

- $60 \mu \mathrm{m}$ pixels

- Asynchronous acquisition of laser returns (not gated)
- Low noise amplification , $0.5 \mathrm{pA} / \mathrm{rt}-\mathrm{Hz}$

- Range and intensity acquired for up to three returns

- Range precision $5 \mathrm{~cm}(0.3 \mathrm{~ns})$

- $60 \mathrm{~Hz}$ operation

\subsection{Packaged SCAs}

Once functioning ROIC die and detector die have been identified, they are "Indium bump hybridized" using the process presently used for IR sensors. Figure 14a shows the hybridized Sensor Chip Assembly (SCA) attached and bonded into a Leadless Chip Carrier and installed in an Integrated Dewar Assembly or IDA. Passive components are installed on the same package to optimized performance.

\section{6² LADAR Receiver in TEC Pkg} Stabilized at Ambient Temperature

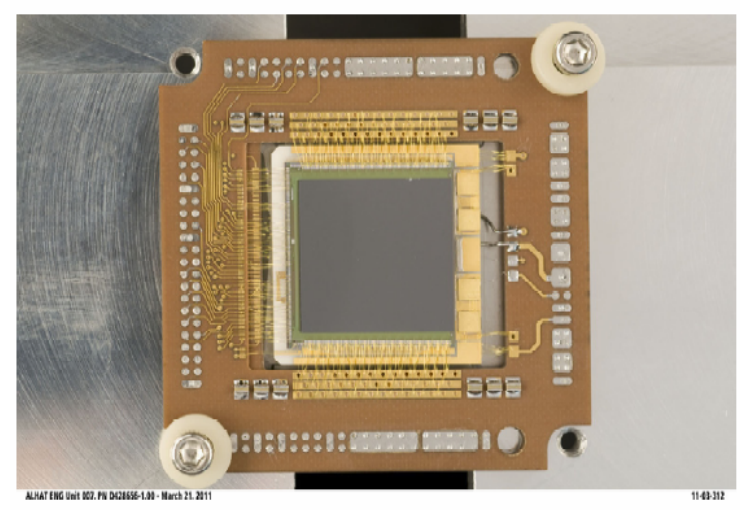

Provides:

-3 Returns: Intensity \& Range

-Asynchronous Detection

-5cm Range Precision
Qualification Test Measures Minimum Detectable Signal (MDS) vs Gain

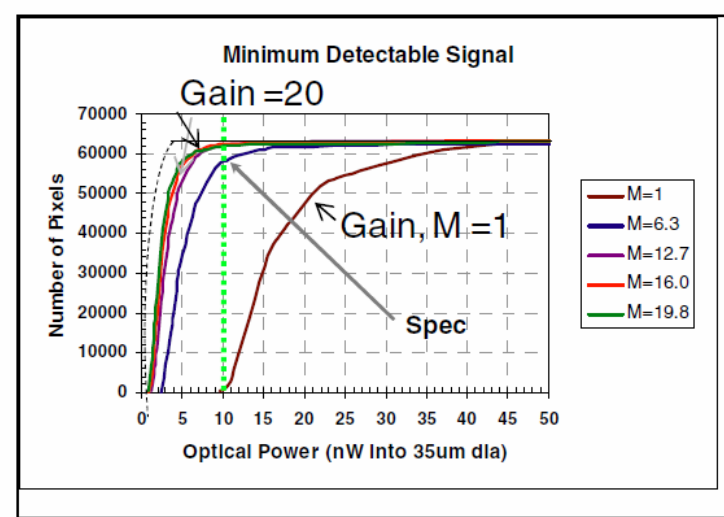

MDS is Arrav Metric - Optical Power for Which $95 \%$ of Pixels Detect $95 \%$ of Time with $1 \%$ FAR -Requires Response/Gain Uniformity -10x Improvement at Gain =20

\section{Delivered to NASA 1Q 2012}

\section{MDS Beats 10nW Performance Specification}

Figure 14. ALHAT SCA Packaged in TEC Stabilized IDA \& MDS Qualification Test Prior to Shipment to NASA

\subsection{SCA \& IDA Test Results}

SCAs are evaluated for full functionality and quantitative measurements are performed across the entire array of key parameters which include signal response, responsivity, noise and NEP. A key sensor/system parameter, Minimum Detectable Signal (MDS) is also determined. SCAs that meet requirements are packaged in a TEC stabilized package, Integrated Dewar Assembly or IDA (Figure 14a) for operation at ambient temperature. Final tests are performed prior to 
delivery. The key test is determining MDS (Figure 14b). It should be emphasized that all data was taken at ambient temperature as was the case for the MMSS scanning arrays.

As discussed in the prior section, Minimum Detectable Signal is a key system-sensor parameter and corresponds to array level signal where high probability of target detection can be achieved. The MDS definition is the signal level at which $95 \%$ of the array triggers on the return laser pulse 95 times or more out of 100 laser shots. Figure 14b plots the cumulative number of pixels which detect the laser pulse 95/100 times as the laser pulse energy is lowered. Operability at high flux is excellent $>98 \%$ at a gain of 20 . MDS is determined as the horizontal axis value when the curve meets the $95 \%$ point we see that the MDS for the array decreases from unity gain value of $45 \mathrm{nW}$ to a $15 \mathrm{nW}$ at a gain of 6 to $\sim 5 \mathrm{nW}$ at a gain of $15-20$, clearly meeting the design goal of $<10 \mathrm{nW}$. Note also that the MDS continues to improve as the gain is increased in contrast with III-V APDs where increases in gain at some point will increase the noise due to excess noise factors. The good performance is due to the near unity excess noise factor for HgCdTe. The solid dark line indicated improvements in performance anticipated for future designs with higher gains. The ALHAT sensor was qualified and shipped to NASA in the 1rst quarter of 2012.

\section{PHOTON COUNTING SENSORS}

\subsection{SCA Design and 2007 Measurements}

Figure 15 illustrates a $4 \times 4$ Photon Counting Sensor, the SB415B. This design and its predecessor, the SB332, use a combination of high gain amplifier and HgCdTe APDs whose design is nearly identical to that used for the previously described scanning and staring arrays operating at ambient temperature. A small APD array is hybridized to the center of the ROIC. Outputs from the APDs operated at moderate gains 50-200 are amplified with low noise GHz amplifiers in each pixel. The output from each channel is buffered and driven off chip at GHz BW by drivers.

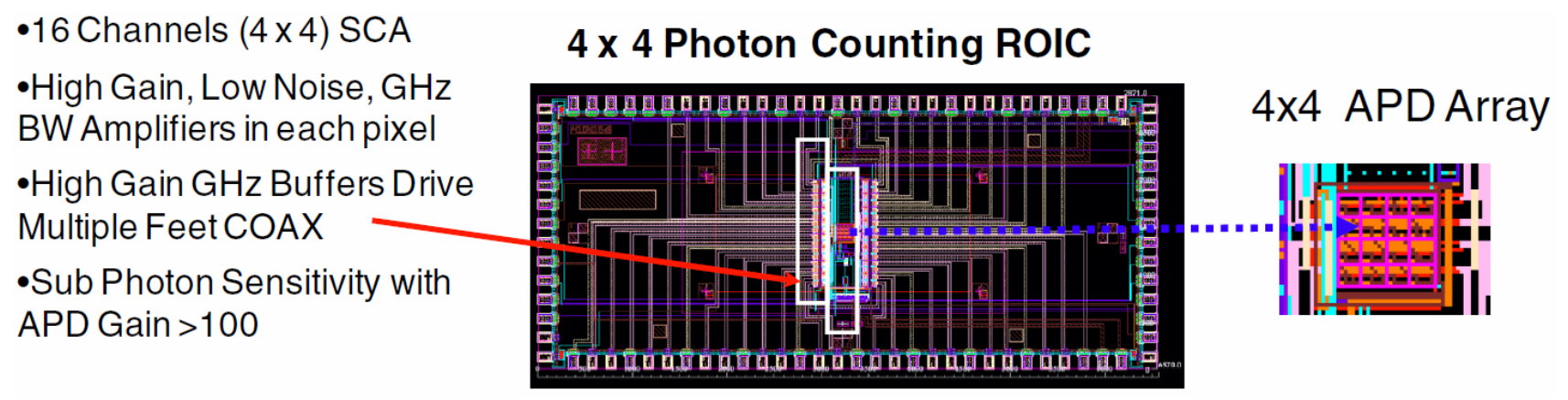

Figure 15. Photon Counting SCA Showing the Small APD Array Hybridized to the GHz BW ROIC

Figure 15 illustrates photon counting achieved in 2007 using the SB332 ROIC design. Measurements were made at an average illumination strength of one photon on the detector per pulse. Figure 1a shows discrimination between zero, one and two photons; $1 \mathrm{~b}$ the ability to detect two closely spaced photons with no afterpulsing and figure 1c shows excellent agreement between the measured and calculated poisson distribution of incoming photons. The detector preserves the photon distribution. 

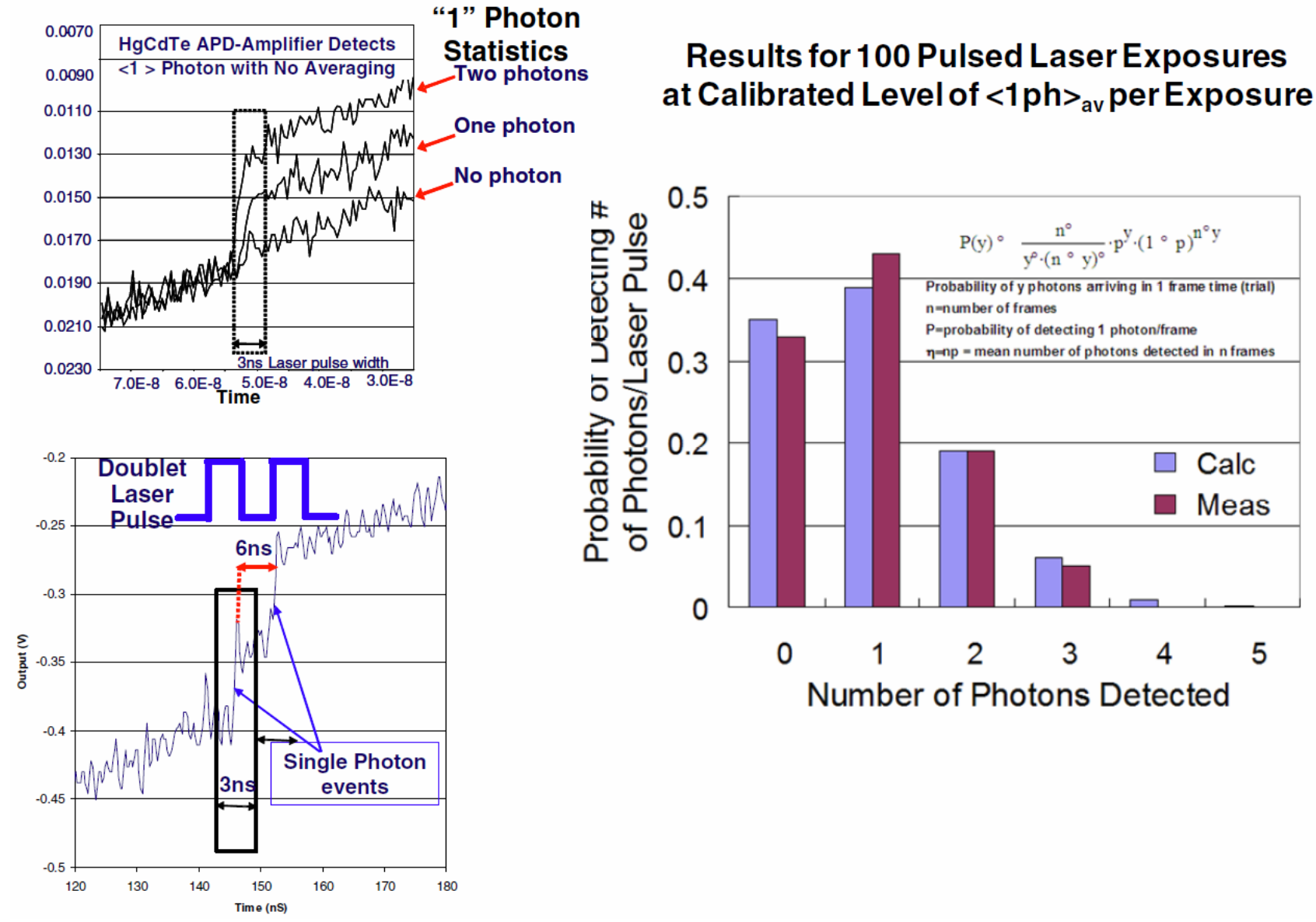

Figure 16. 2007 Demonstration of Photon Counting: (a) Multiple acquisitions showing detection of 0,1 and 2 photons with average illumination of $<1>$ photon (b) Acquisition of closely double pulse closely spaced photons ( $<6 \mathrm{ns)} \mathrm{with} \mathrm{no}$ afterpulsing observed; (c) Comparison of measured photon distribution for 100 pulsed laser exposures vs predicted from Poisson distribution showing good agreement

\subsection{Breakthroughs in Performance - ROSES Program}

Under NASA's ROSES program (contract with University of Hawaii) a second generation ROIC, SB415 was integrated with APD arrays fabricated using a process optimized for low surface and bulk dark current. Because of the GHz amplifiers it was suspected that one contribution to residual dark counts was glow (light emitted due to hot carriers) from the $\mathrm{GHz}$ bandwidth amplifiers. High sensitivity imaging at the silicon bandedge of the ROIC was performed by the $\mathrm{U}$ of $\mathrm{H}$ team using their ultra low light leakage $(<1$ photon/hour) cryo-test station, Figure 18 . The results (Figure 17) clearly shows the correlation between location of the GHz buffers and emitted light from the photo-micrograph on the left. The graph on the right shows the reduced light output when bias was adjusted to minimize glow while achieving required performance. Detection of an individual photon is shown in Figure 19a. Signal to noise $>10$ with low dark counts is achieved. The curve shows very low sub pico-amp surface dark current (seen as shallow slope of the curve). This low surface dark current enables look times as long as $10 \mathrm{~ms}$ without resetting. Performance parameters of the SCA are summarized in Figure 19b. The dark counts and SNR taken together enable a probability of detection $>95 \%$ with low FAR $<1 \%$. Units were shipped to $\mathrm{U}$ of $\mathrm{H} 4^{\text {th }}$ quarter of 2010 . Photon counting was verified by researchers at $\mathrm{U}$ or $\mathrm{H}$ using the cryo-system shown in Figure 18. Typical results are shown in Figure 20. Two channels are monitored simultaneously and show 0,1 and 2 photons. 
-16 Channels (4 x 4) SCA 4 x 4 Photon Counting ROIC

•High Gain, Low Noise GHz

Amplifiers in each pixel

-High Gain GHz Buffers

Drive Multiple Feet COAX

-Sub Photon Sensitivity with APD Gain >100

GHZ Output Buffers Imaging of ROIC During Operation at Si Band-edge

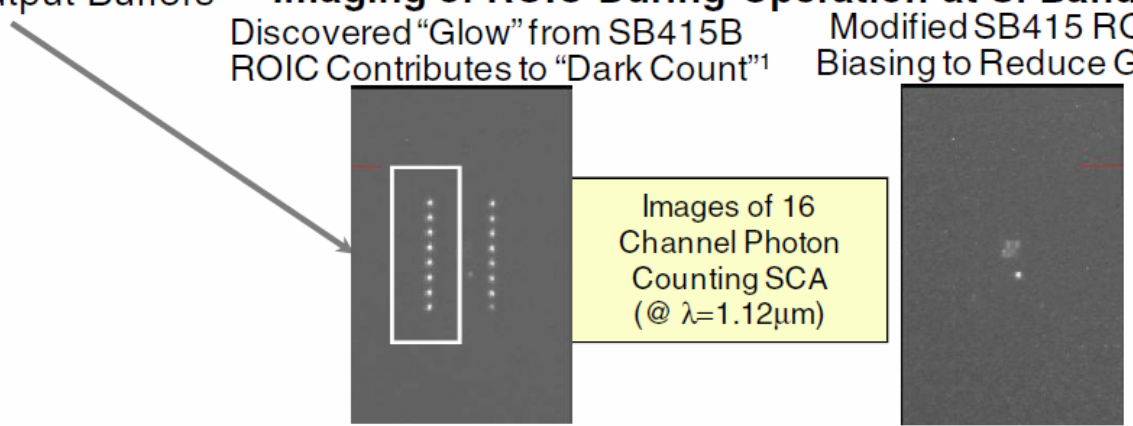

Effective Glow Rate 1E7ph/s/amp Bias Optimized Reduces Glow Rate By >1000X

Glow Minimized by Optimum Biasing Reduces SCA DCR to $<10^{4}$

\section{Optimized SCAs Delivered to U of H Under NASA's ROSES Program 4Q 2010}

Figure 17. Glow From Silicon ROIC is a Significant Contributor to Dark Count Rate (a) ROIC Layout with two banks of $\mathrm{GHz}$ amplifiers on either side of the detector array, (b) Long integration time image of ROIC in operation quantifying the "glow" flux at about 1E7 photons per second; (c) Optimizing biases reduces glow illumination to 1E5 ph/s.

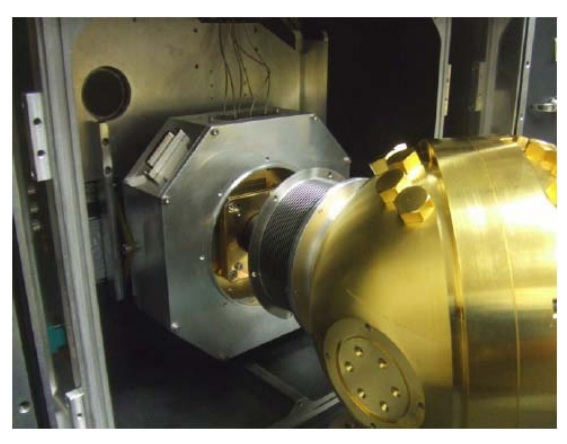

(a)

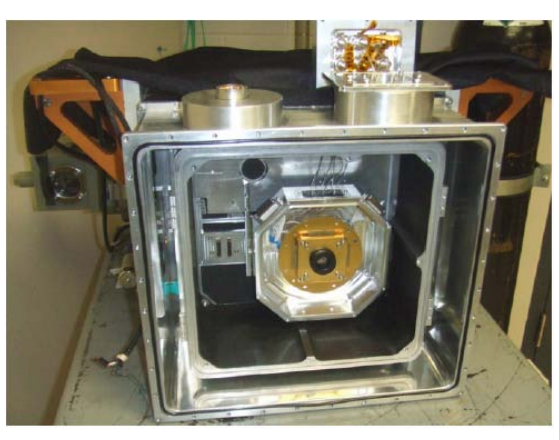

(b)

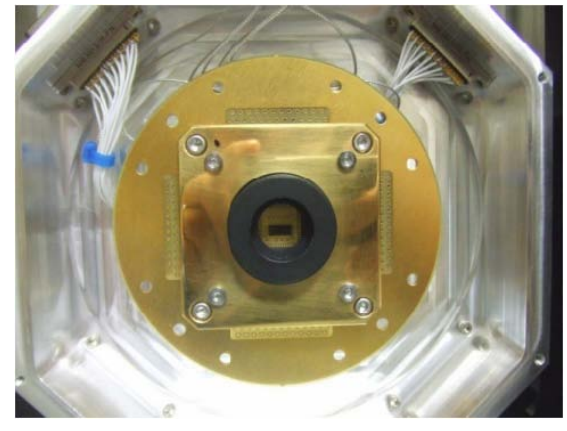

(c)

Figure 18. Cryogenic Low Light Test Facility at University of Hawaii (a) Light Tight Facility Showing Sample Holder and Connection to Controlled Illumination Sources; (b) Ssmple Holder with SB415 Mounted; (c) Close up of Sample Holder with SB 415B 


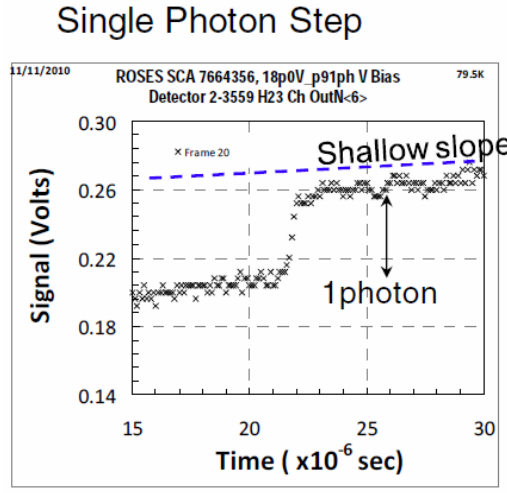

- Single Photon Step is $\sim 1 / 100^{\text {th }}$ of dynamic range with good $\mathrm{SNR}>10$

- Low Surface Dark Current (Slope) enables Tint to $>10 \mathrm{~ms}$ without reset
Parameters

\begin{tabular}{|c|c|}
\hline APD Design & Improved B Design \\
\hline Process & $\begin{array}{l}\text { Improved Clean } \\
\text { Surface Preparation }\end{array}$ \\
\hline Operating Voltages & Vbias Det $<20 \mathrm{~V}$ \\
\hline Operating Temperature (K) & $80-180$ or greater \\
\hline Maximum Gain & $200-350$ \\
\hline $\begin{array}{l}\text { Dark Count Rate (DCR) } \\
\text { (counts/sec) at M>100 }\end{array}$ & $\begin{array}{c}<10^{4}(80 \mathrm{~K}-160 \mathrm{~K})^{1} \\
<10^{5} 180 \mathrm{~K}\end{array}$ \\
\hline Surface Dark Current (Amp.) & $<1 \mathrm{E}-13$ \\
\hline Max Tint w/o Reset (ms) & 10 \\
\hline Operability & $>90 \%$ \\
\hline Probability of Detection & $>95 \%$ \\
\hline False Alarm Rate ${ }^{2}$ & $<1 \%$ \\
\hline
\end{tabular}

${ }^{1}$ Limited by Residual Glow, ${ }^{2}$ Meas Over $>1 \mu$ s interval

\section{Good SNR \& $<10^{4}$ DCR Provides $>95 \%$ PD with $<1 \%$ FAR}

Figure 19. (a) Single Photon Measurement Showing Good SNR ratio, at Low Dark Count Rate and Low Surface Leakage Current (Slope) and 100x Dynamic Range (b) Summary of APD Performance Parameters

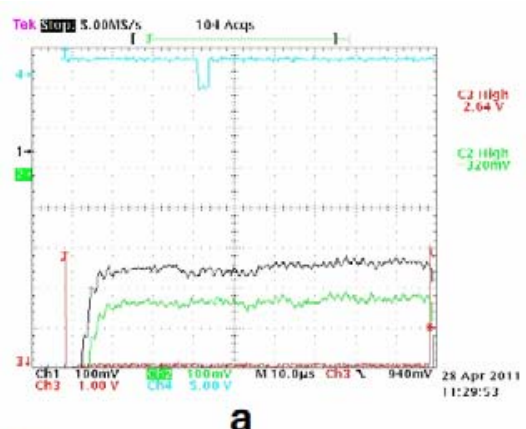

a

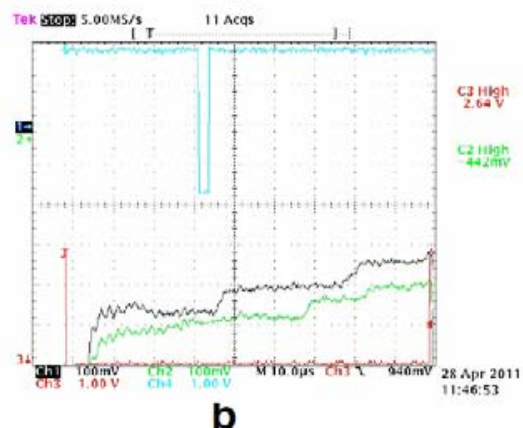

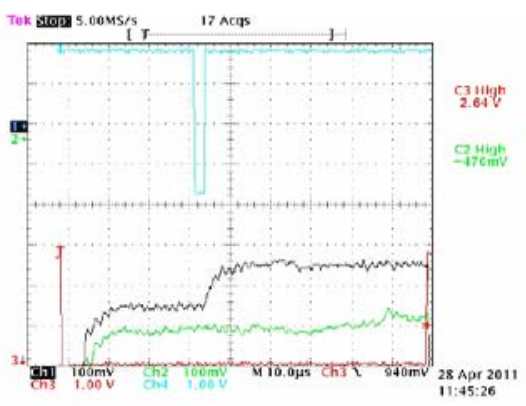

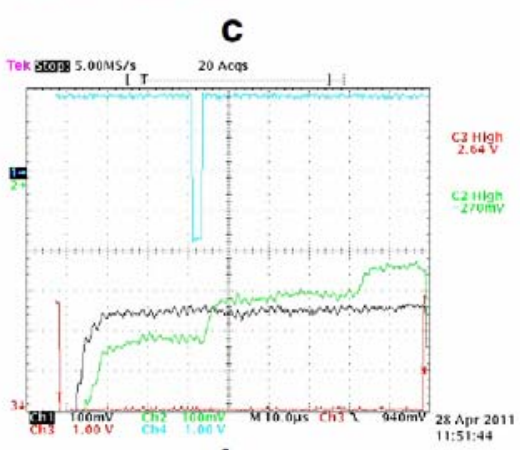

d

Figure 20. Validation of Photon Counting by the U of H Team. Examples of two (\#6 black and \#11 green) of the sixteen output channels of an SB415B during 90 sec exposures illuminated by a $0.4 \mathrm{~m}$ sec IR LED pulse (blue trace) averaging one photon per pixel. Panel a shows no photon events, b shows a double photon event on channel \#11, c shows a single photon on channel \#6 and, d shows a single photon on channel \#11. Single photon events not coinciding with the LED pulse are attributed to readout glow at the measured rate of 104 photons/sec i.e. one event per 100usec. 


\section{FUTURE DIRECTIONS}

Figure 21 illustrates future direction envisioned for linear mode photon counting. Here we show operational $256 \times 256$ arrays linear mode SCAs developed for NASA Langley under the ALHAT program. As previously discussed these APD arrays have demonstrated excellent uniformity at a single bias voltage with gains up to 100 at ambient and SCAs at ambient temperature have shown excellent uniformity with $>99 \%$ operability and Minimum Detectable Signal (MDS) $<10 \mathrm{nW}$. The architecture of the $4 \times 4$ linear mode photon counting SCAs which as discussed has been realized with very low dark count rates can readily be scaled to these larger formats.

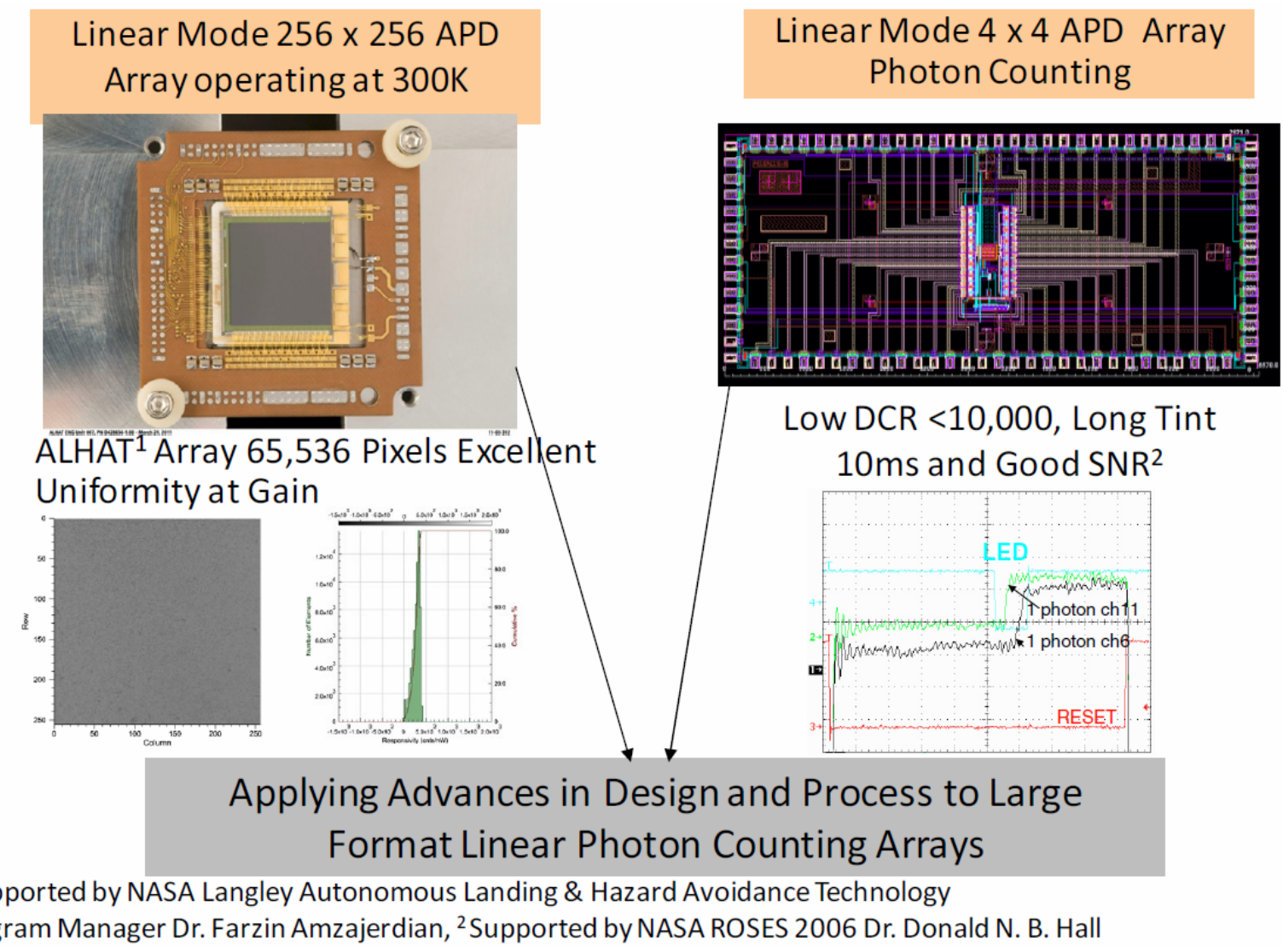

Figure 21. Future Directions in Linear Mode Photon Counting. Advances in low dark current linear mode photon counting can readily be applied to large format configurations such as the $256 \times 256$ ALHAT LADAR receiver developed for NASA which at ambient temperature have demonstrated excellent uniformity with a single APD bias, high gains to 100 and excellent sensitivity with MDS <10 nW.

\section{SUMMARY}

High performance Scanning, Staring and Photon Counting LADAR receivers have been developed using high performance common elements specifically: HgCdTe APDs engineered for an excess noise of $1\left(\mathrm{~F}_{\mathrm{ex}} \sim 1\right)$ both at ambient temperatures integrated with deep submicron ROIC integrated circuits whose design enables asynchronous (non gated) capture of multiple up to three laser returns. Large format scanning $256 \times 4$ and $256 \times 256$ staring receivers built with these common elements have been integrated into common compact TEC packaging, tested and delivered to Navy and NASA customers respectively. Qualification testing has shown operability $>99 \%$ with low Minimum Detectable Signal (MDS) $<10 \mathrm{nW}$ at $300 \mathrm{~K}$. The same APD/circuit technology has shown photon counting with SNR $>10$ to above $180 \mathrm{~K}$ with dark count rates still limited by Silicon Circuit "glow" at $<10^{4}$ cps below $160 \mathrm{~K}$. In the future, the architecture of the $4 \times 4$ linear mode photon counting SCAs realized with very low dark count rates can readily be scaled to larger formats. The use of common designs in HgCdTe APD arrays with high gain and no excess noise at ambient, and low temperature coupled with mature high yield ROICs and compact TEC packaging enables an affordable new capability for high acuity $3 \mathrm{D}$ real-time imaging, long range threat detection, and navigation. 


\section{ACKNOWLEDGEMENTS}

The authors wish to acknowledge the following scientists, technicians and researchers who have contributed to and supported the development of advanced LADAR sensors at Raytheon Vision Systems (Figure 22).

- Raytheon Vision Systems; Goleta, CA

- G. Chapman

- G. Crawford

- E. Herrin

- C. Hoyt

- V. Lee

- K. Liu

- M. Mitani

- W. McKeag

- T. Veeder

- J. Wehner

- Raytheon Missile Systems; Tucson, AZ

- T. Roberts

- C.T. Robinson

- J. Neisz

- R. Rinker

- C. Andressen

Figure 22. Acknowledgements
- HRL Labs, LLC; Malibu, CA

- T. DeLyon

- NASA Goddard Space Flight Center; Greenbelt, MD

- H. Moseley

- B. Rauscher

- X.Sun

- NASA, Langley; Hampton, Virginia

- F. Amzajerdian

- University of Hawaii, IFA; Hilo, Hawaii - K. Hodapp

- F. Hee 